Oncol Res Treat 2015;38:51-55

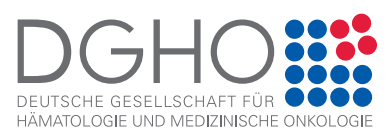

Deutsche Gesellschaft für Medizinische Hämatologie und Onkologie e.V.

DGHO Hauptstadtbüro

V.i.S.d.P. Michael Oldenburg

Tel. 030 / 2787 6089-0, Fax: -18

oldenburg@dgho.de

www.dgho.de

\title{
Deutsche Stiftung für junge Erwachsene mit Krebs gegründet
}

\section{Neue Perspektiven für junge Patienten}

Junge Erwachsene mit Krebs benötigen eine spezielle medizinische Behandlung und psychosoziale Versorgung. Die neu gegründete Deutsche Stiftung für junge Erwachsene mit Krebs will die Therapiemöglichkeiten junger krebskranker Frauen und Männer verbessern. Zudem widmet sie sich dem Aufbau von Versorgungsstrukturen, mit denen spezifische Probleme dieser Patientengruppe besser adressiert werden können. Des Weiteren will die Stiftung dazu beitragen, dass die Forschung in diesem Problembereich intensiviert wird.

Nur etwa 3,2 Prozent (ca. 15 000) aller neu diagnostizierten Krebspatienten in Deutschland ist zwischen 15 und 39 Jahre alt. Dennoch benötigt diese Patientengruppe sowohl in der Behandlung als auch in der Nachsorge besondere Aufmerksamkeit. «Bei jungen Erwachsenen kommt die Diagnose Krebs zu einer Zeit, in der Gedanken an Krankheit, Sterben und Tod normalerweise keinen Platz haben», betont Dr. Karolin Behringer von der Onkologischen Ambulanz des Universitätsklinikums Köln und Leiterin der Arbeitsgruppe «Survivorship» in der Deutschen Hodgkin Studiengruppe (GHSG). Die Herausforderung für Ärzte und Betreuer, so Behringer, besteht darin, Verständnis für das Autonomiebedürfnis und für die besonderen Wünsche und Sorgen dieser Patienten aufzubringen, dabei aber gleichzeitig die rasche und planmäßige Durchführung einer notwendigen und oft intensiven Therapie sicherzustellen.

\section{Bessere Versorgung und Nachsorge als wichtigstes Stiftungsziel}

«Die spezifischen Probleme, vor denen junge Krebspatienten und die sie behandelnden Ärzte im heutigen Versorgungssystem stehen, waren die entscheidende Triebfeder für die Gründung der Deutschen Stiftung für junge Erwachsene mit Krebs», betont Prof. Dr. Mathias Freund, Vorsitzender des Stiftungskuratoriums und Geschäftsführender Vorsitzender der DGHO Deutsche Gesellschaft für Hämatologie und Medizinische Onkologie e.V.. «Bei jungen Erwachsenen mit schweren Krebserkrankungen führen wir sehr eingreifende Therapien durch. Dabei ist es tragisch, wenn die Patientinnen und Patienten, die im Aufbruch ins Leben stehen, in ihrer Lebensplanung außerordentlich beeinträchtigt sind und im späteren Leben häufig weniger gute Chancen haben als die altersgleichen Gesunden. Die bestehenden Unterstützungsangebote reichen aus unserer Sicht nicht aus. Hier sind wir einfach verpflichtet, etwas zu tun.»

Ein wesentliches Ziel der neuen Stiftung ist die Verbesserung der Versorgungsstrukturen beispielsweise durch die Realisierung von Projekten, mit denen sowohl die medizinische und psychosoziale Versorgung als auch die Nachsorge von jungen Krebspatienten verbessert werden können. «Geplant sind konkrete Sprechstunden, die sich speziell mit Fragen der Langzeittoxizität von Anti-Tumor-Therapien befassen», so Freund. Auch bundesweite Programme zur Armutsprävention und zur Unterstützung bei der Wiedereingliederung ins Berufsleben sind denkbar. «Es muss dringend etwas getan werden: Zwar liegen die Krebserkrankungen bei jungen Erwachsenen nur 3,2 Prozent der neuen Diagnosen. Die Hei-

\begin{tabular}{ll}
\hline KARGER & ๑ 2015 S. Karger GmbH, Freiburg \\
Fax +49 7614520714 & Accessible online at: \\
$\begin{array}{l}\text { Information@Karger.com } \\
\text { www.karger.com }\end{array}$ & www.karger.com/ort
\end{tabular}


lungsrate liegt jedoch bei 80 Prozent. Auf diese Weise sind in 20 Jahren etwa 250000 Menschen zu Überlebenden nach Krebs geworden, mit all ihren Problemen», ergänzt Freund.

\section{Von Chemo-Brain bis Kinderwunsch}

Gerade die Langzeittoxizität intensiver Therapiemaßnahmen ist für Menschen, die sich als junge Patienten einer Krebstherapie unterzogen haben, ein zentrales Problem: «Junge Menschen mit Krebs sind «Langzeit-Überlebende», und sie sind neben an Krebs erkrankten Kindern am längsten mit den negativen Folgen der Anti-Tumor-Therapien konfrontiert», betont Prof. Dr. Volker Diehl, Gründer der Deutschen Hodgkin-Studiengruppe und ebenfalls Mitglied des Stiftungskuratoriums. So stellen etwa die kognitiven Folgen der Chemo-/Radiotherapie, auch «Chemo-Brain» genannt, gerade für junge Akademiker in der Langzeitperspektive ein mögliches Problem dar.

«Gerade bei den jungen Erwachsenen, wenn die Familienplanung im Vordergrund steht, sind die Folgen einer zytotoxischen Therapie auf Libido, Sexualität und Kinderwunsch häufig gravierend», so Diehl. Ärzte, die nicht regelmäßig mit Krebspatienten im jungen Erwachsenenalter zu tun haben, sind hier nicht selten in der Arzt-Patienten-Kommunikation überfordert.

Dabei schließen sich Krebserkrankung im jungen Alter und Kinderwunsch heute häufig nicht mehr aus: «Wir verfügen über eine Reihe von Möglichkeiten, die Fruchtbarkeit zu erhalten, beispielweise die Kryokonservierung, also das Einfrieren von Eizellen. Entsprechende Informationen sollten ein wesentlicher Inhalt des Arzt-Patienten-Gesprächs sein. Nur dann können fertilitätserhaltende Maßnahmen frühzeitig eingeleitet werden», betont Behringer.

\section{Wer ist zuständig?}

Beitragen möchte die Deutsche Stiftung für junge Erwachsene mit Krebs auch zu einer notwendigen Diskussion über die Zuständigkeiten und Anlaufstellen für junge Patienten mit Krebserkrankungen. «Um den Patientinnen und Patienten wirklich gerecht zu werden, brauchen wir ein Netzwerk besonderer «kompetenter Anlaufstellen» in Deutschland, in denen pädiatrische Onkologen und Erwachsenenonkologen, aber auch andere Berufsgruppen wie beispielsweise Psychologen oder Sozialarbeiter eng miteinander kooperieren», betont Diehl. Darüber hinaus können diese Anlaufstellen auch von Hausärzten genutzt werden. Denn häufig werden Hausärzte von Patienten nach einer schon länger zurückliegenden erfolgreichen Krebsbehandlung wegen der Spätfolgen aufgesucht. «Hier», so Diehl, «bedarf es einer intensiven Schulung von Allgemeinmedizinern durch die Tumorzentren und Fachgesellschaften.»

\section{Beitrag zu Forschung, Aus- und Fortbildung}

Ein weiteres Ziel der Deutschen Stiftung für junge Erwachsene mit Krebs ist, die Erforschung der spezifischen medizinischen und psychosozialen Probleme von Adoleszenten mit Krebs deutlich zu intensivieren. «Wir wissen beispielsweise noch längst nicht genug darüber, wie wir bei einer Krebsbehandlung, speziell bei jungen Erwachsenen, die optimale Balance zwischen Heilungschancen und unerwünschten Wirkungen herstellen können. Natürlich wollen wir möglichst viele Patienten heilen, aber gleichzeitig ist es auch unser Ziel, die negativen Folgen einer intensiven medikamentösen Therapie oder Strahlenbehandlung zu minimieren», so Freund.

Schließlich muss jungen Erwachsenen mit Krebs im Rahmen der Ausbildung von Studenten und besonders Krebsfachärzten sowie nachbetreuenden Ärzten mehr Aufmerksamkeit als bisher gewidmet werden. Auch dazu möchte die Deutsche Stiftung für junge Erwachsene mit Krebs beitragen. Unter anderem will sie medizinische Einrichtungen bei der Ausbildung und der Fort- und Weiterbildung von entsprechendem Fachpersonal unterstützen. Darüber hinaus sollen Internet-«Chatrooms» für junge Krebspatienten initiiert und gefördert werden, in denen diese sich austauschen, helfen und vielleicht sogar als «Paten» für andere fungieren können.

Krebs erforschen. Zukunft spenden. Gemeinsam mit Ihnen!

Die Arbeit der Deutschen Stiftung für junge Erwachsene mit Krebs wird ausschließlich durch Spenden finanziert und erfolgt auf ehrenamtlicher Basis. Aus diesem Grund ist jede - noch so kleine - Spende wichtig. Durch Ihre Spende helfen Sie uns bei der Förderung von Forschungsprojekten, beim Aufbau eines Netzwerks von Spezialistinnen und Spezialisten oder bei der Weiterentwicklung der Aus-, Fort- und Weiterbildung. Damit unterstützen Sie uns, jungen Menschen mit Krebs zu helfen.

Kontoverbindung der Deutschen Stiftung für junge Erwachsene mit Krebs:

Postbank, IBAN: DE57 1001001008342261 04, BIC: PBNKDEFF 


\section{Monatsthema im Februar: Klinische Studien in der Krebstherapie - Informationen für Patienten}

Für Krebspatienten ist die Teilnahme an einer Studie mit der Chance verbunden, von neuen Therapieverfahren oder Arzneien zu profitieren, die im normalen klinischen Alltag noch nicht zugänglich sind. Doch vor einer Studienteilnahme stellen sich Patienten viele Fragen. Antworten bietet das ONKO-Internetportal im Februar mit einem Leitfaden für Krebspatienten, die sich für eine Studienteilnahme interessieren.

Mit enormem Aufwand wird heute an der Entwicklung neuer Behandlungsmöglichkeiten von Krebserkrankungen gearbeitet. Bis zur Zulassung eines neuen Medikaments vergehen oftmals 10 Jahre. Klinische Studien stehen am Ende dieser Entwicklungsphase und sind für die Zulassung von neuen Medikamenten unumgänglich.
Der Vorteil einer Studienteilnahme liegt für Patienten im frühzeitigen Zugang zu innovativen Behandlungen oder Verfahren, die kurz vor der Zulassung noch nicht auf normalem Weg erhältlich sind, dem Betroffenen aber möglicherweise sehr helfen können. Neue Methoden bergen viele Chancen, können aber auch bisher unbekannte Risiken und Nebenwirkungen haben oder weniger wirksam sein als herkömmliche Verfahren. Dies gilt es zu bedenken.

Doch wie finden Patienten überhaupt eine passende Studie? Welche Zugangsvoraussetzungen müssen erfüllt werden? Was sollten Patienten vor einer Teilnahme unbedingt wissen? Diese und weitere Fragen werden im Monatsthema Februar der ONKO-Internetportals unter www.krebsgesellschaft.de/ thema_februar2015 beantwortet. 


\section{Curt Meyer Gedächtnis-Preis 2014: Jane Holland vom MDC erhält Auszeichnung für Arbeit zum basalen Brustkrebs}

\section{Berliner Krebsgesellschaft würdigt Entdeckung von neuen therapeutischen Angriffspunkten bei tripel-negativen Brusttumoren}

Am 10. Dezember 2014 hat die Berliner Krebsgesellschaft den Curt Meyer Gedächtnis-Preis 2014 verliehen. Die mit 10 000 Euro dotierte Auszeichnung ging an Dr. Jane Holland vom Max-Delbrück-Centrum für Molekulare Medizin (MDC) Berlin-Buch für ihre wegweisende Arbeit zum basalen Brustkrebs. «Jane Holland hat mit ihrer Arbeit zu einem besseren Verständnis über den basalen Brustkrebs beigetragen und damit Grundlagen für die Entwicklung neuer Therapieoptionen gelegt», begründet Prof. Dr. Dr. Peter M. Schlag im Vorfeld die Auszeichnung der 34-jährigen Australierin, die seit 2007 am MDC über Brustkrebs forscht. «Diese Leistung wollen wir mit dem Curt-Meyer Gedächtnispreis 2014 würdigen.» Die Ergebnisse waren im Dezember 2013 im Fachmagazin «Cell Reports» erschienen und hatten in der Fachwelt für große Aufmerksamkeit gesorgt.

Basaler Brustkrebs ist eine schnell wachsende und aggressive Krebsart und geht tendenziell mit einer schlechten Prognose einher. In der Regel handelt es sich um tripel-negative Tumore, die so heißen, weil sie weder Rezeptoren für die Hormone Östrogen oder Progesteron noch Rezeptoren für den Wachstumsfaktor Her2 besitzen. Da die Tumore nicht auf Antihormontherapien oder die Antikörpertherapie gegen Her2 ansprechen, ist die Chemotherapie bislang die einzige medikamentöse Therapieoption.

Um die Suche nach neuen Therapiemöglichkeiten voranzutreiben, haben Holland und ihre Kollegen vom MDC ein genetisches Mausmodell entwickelt und dabei gleich mehrere bemerkenswerte Entdeckungen gemacht. «Wir konnten erstmals zeigen, dass der Chemokinrezeptor CXCR4 beim basalen Brustkrebs eine entscheidende Rolle spielt», beschreibt Jane Holland den neuen Fund. «Damit haben wir neben den beiden anderen Krebstreibern WNT und Met eine dritte Komponente identifiziert, die diesen Tumor so aggressiv macht.»

Und nicht nur das: In Tests mit verschiedenen Hemmstoffen zeigte sich, dass das Chemokinsystem und die beiden Signalwege Wnt/beta-Catenin und HGF/Met auch ideale therapeutische Angriffspunkte sind. Die eingesetzten Hemmstoffe werden bereits bei anderen Krebsarten erprobt, kommen aber bislang nicht beim Brustkrebs zum Einsatz. Mit dem dreifachen Angriff gelang es den Wissenschaftlern, das Krebswachstum bei den Mäusen für mehrere Wochen zu unterdrücken. «Es hat sich gezeigt, dass die Dreifach-Blockade am wirksamsten ist», sagt Holland. «Das macht uns große Hoffnung, dass der basale Brustkrebs eines Tages vielleicht doch zielgerichtet behandelt werden kann, so wie es bei anderen Brustkrebserkrankungen bereits der Fall ist.» In einer Kooperation mit der Charité will Holland die Hemmstoffe demnächst am menschlichen Gewebe weiter testen. Vorerst allerdings noch im Labor.

Die Preisträgerin Dr. Jane Holland hat an der Universität in Adelaide (Australien) Chemie und Mikrobiologie studiert. Dort promovierte sie im Jahr 2006 mit einer Arbeit über Brustkrebs. Seit 2007 ist sie Senior Postdoktorandin in der Forschungsgruppe «Signalvermittlung in Entwicklung und Krebsentstehung» von Prof. Walter Birchmeier am MDC. 


\section{BNHO}

Berufsverband der Niedergelassenen

Hämatologen und Onkologen

in Deutschland e.V.

BNHO e.V.

Geschäftsstelle

PD Dr. Stephan Schmitz, Vorsitzender

Armin Goetzenich, Geschäftsführer

Vor den Siebenburgen 2, 50676 Köln, Germany

Tel. +49 221998 798-0, Fax -22

info@bnho.de

www.bnho.de

\section{Neue Initiative von BNHO und IKK gesund plus: Onkologische Zweitmeinung mit Qualitätssiegel}

Zweitmeinungen können bei Patienten mit onkologischen Erkrankungen das Vertrauen in die Anti-Tumor-Therapie stärken und dazu beitragen, ein optimales Behandlungskonzept zu erstellen. Allerdings muss eine hohe Qualität der Zweitmeinung gewährleistet sein. Der Berufsverband der Niedergelassenen Hämatologen und Onkologen in Deutschland - BNHO e.V. kooperiert ab sofort mit der IKK gesund plus aus Sachsen-Anhalt, um eine qualitativ hochwertige onkologische Zweitmeinung innerhalb der GKV zu ermöglichen.

Das Einholen von Zweitmeinungen ist in der Medizin nichts Neues: Schon immer haben Ärzte, die sich bei einer Diagnose oder Therapieentscheidung unsicher fühlten, bei Kollegen mit spezifischer Expertise um Rat gefragt. Und schon immer gab es Patienten, die mehr als nur einen Arzt aufgesucht haben, bevor sie sich mit einer vorgeschlagenen Behandlung einverstanden erklärten. In Zeiten zunehmender Qualitätsdiskussionen im Gesundheitswesen und nicht zuletzt auch dank des Internets haben ärztliche Zweitmeinungen in den letzten Jahren deutlich an Popularität gewonnen. Hinter vielen Angeboten stecken oftmals kommerzielle Interessen.

\section{Onkologische Zweitmeinung auf Kassengutschein}

Mit einer neuen Projektkooperation wollen der Berufsverband der Niedergelassenen Hämatologen und Onkologen in Deutschland - BNHO e.V. und die Krankenkasse IKK gesund plus aus Sachsen-Anhalt jetzt dafür sorgen, dass Patienten mit hämatologischen oder onkologischen Erkrankungen auf Wunsch eine Zweitmeinung erhalten, bei der die Qualität stimmt und die Finanzierung geregelt ist. «Die Einbindung des BNHO ist dabei eine Art Qualitätssiegel für die Zweitmeinung», erläutert der BNHO-Geschäftsführer Armin Goetzenich. «Unsere Mitglieder sind ausgewiesene Krebsspezia- listen mit langjähriger Erfahrung in der Behandlung und emotionalen Begleitung von Krebspatienten. Niemand ist besser geeignet, eine qualifizierte Zweitmeinung zu erstellen, die den Patienten auch wirklich weiterhilft.» Herausragendes Element der neuen Projektkooperation ist, dass die niedergelassenen Krebsspezialisten die Zweitmeinung nicht auf Basis der Patientenakte erstellen, sondern im Rahmen der persönlichen Vorstellung und Untersuchung.

\section{Kooperation bringt Vorteile für alle Beteiligten}

«Aus unserer Sicht ist die Projektkooperation mit dem BNHO optimal», betont Uwe Schröder, Vorstand der IKK gesund plus. «Zweitmeinungen werden insbesondere von Krebspatienten zunehmend nachgefragt. Als IKK gesund plus können wir Patienten mit Krebserkrankungen bei entsprechenden Anfragen jetzt ein attraktives und vor allem qualitativ hochwertiges Serviceangebot machen. Dadurch wird sich die Zufriedenheit der Patienten mit der Versorgung verbessern, und gleichzeitig tragen wir bei den oft sehr aufwändigen Krebstherapien zu einer besseren Versorgungsqualität bei.»

Aus Sicht der Patienten wird durch die Projektkooperation von BNHO und IKK gesund plus nicht nur die Erstattung der Kosten für die Zweitmeinung klar und unbürokratisch geregelt. Die «Zweitmeinung mit BNHO-Siegel» erspart den Patienten auch eine zeitraubende Suche nach entsprechenden Experten und schützt vor Anbietern mit kommerziellem Interesse. Politisch werden BNHO und IKK gesund plus mit ihrer Initiative zu einem Trendsetter: Das Versorgungsstärkungsgesetz, das sich derzeit in der politischen Abstimmung befindet, soll ein Recht auf Zweitmeinung im Gesetz verankern. Die Details regelt der Gemeinsame Bundesausschuss. «Bis das so weit ist, werden sicherlich von unserem Projekt schon viele Krebspatienten profitiert haben», so Goetzenich. 